\title{
Causas de extracción dental en un centro de salud de la sierra de Zongolica
}

\author{
MSP. Jaime Jaramillo Vázquez \\ jaimejaramillo09@hotmail.com \\ https://orcid.org/0000-0003-4465-8720 \\ Servicios de Salud de Veracruz \\ *Dr. Javier Salazar Mendoza \\ jasalazar@uv.mx \\ https://orcid.org/0000-0001-9172-8731 \\ Universidad Veracruzana, Facultad de Enfermería Orizaba \\ C.D. Teresita Margarita Silva Díaz \\ margaritasilva21@ hotmail.com \\ https://orcid.org/0000-0002-6958-4871 \\ Servicios de Salud de Veracruz \\ M.C.Enf. María de los Ángeles Onofre Santiago \\ aonofre@uv.mx \\ https://orcid.org/0000-0002-8951-4379 \\ Universidad Veracruzana, Facultad de Enfermería Orizaba \\ MSP. Sergio Rodríguez García \\ sergio.rg@icest.mx \\ https://orcid.org/0000-0003-0985-8508 \\ Servicios de Salud de Veracruz \\ Instituto de Ciencias y Estudios Superiores de Tamaulipas A.C \\ C.D. Osvaldo Cruz Onofre \\ osvy.co92@gmail.com \\ https://orcid.org/0000-0002-3937-1664
}

\section{RESUMEN}

Introducción: la extracción de órganos dentarios es uno de los procedimientos que se realiza con mayor frecuencia en un consultorio de Odontología, varios factores influyen en que esto suceda, se ha demostrado que la caries dental y enfermedades periodontales son las principales causas de pérdida de dientes. El procedimiento consiste en separar la articulación alveolo dentaria que está formada por encía, hueso, diente y periodonto desgarrando el periodonto en su totalidad. Objetivo: determinar las causas de extracción de órganos dentales en el servicio de Odontología de un Centro de Salud de la Sierra de Zongolica, en el periodo enero 2015 diciembre 2019. Estrategias metodológicas: 
estudio cuantitativo descriptivo y trasversal, que incluyó las extracciones de órganos dentales que se realizaron por el servicio de Odontología en un Centro de Salud de la Sierra de Zongolica que pertenece al municipio de Astacinga. Resultados: se realizaron 599 extracciones, 236 primarios y 363 permanentes en 483 usuarios de las que 305 fueron mujeres lo que representó el 63.1\%. Discusión: la salud bucal como parte integral de la salud general aún se encuentra lejos, se sigue priorizando la actividad curativa en lugar de actividades preventivas, en los resultados se puede observar que las personas llegan tardíamente a consulta odontológica con el diagnóstico de raíz dental retenida. Conclusión: los primeros molares inferiores en la dentición permanente fueron los más afectado mientras que los incisivos centrales anteriores superiores en los órganos primarios.

Palabras claves. (DeCS): extracciones dentales; frecuencia; causa; órganos dentales. 


\title{
Causes of dental extraction in a health center in the sierra de Zongolica
}

\begin{abstract}
Introduction: the extraction of dental organs is one of the procedures that is most frequently performed in a dentistry office, several factors influence this to happen, it has been shown that tooth decay and periodontal diseases are the main causes of tooth loss. The procedure consists of separating the dental alveolus joint, which is made up of the gum, bone, tooth and periodontium, tearing the periodontium in its entirety. Objective: determine the causes of extraction of dental organs in the Dentistry service of a Health Center of the Sierra de Zongolica, in the period January 2015 to December 2019. Methodological strategies: descriptive and cross-sectional quantitative study, which included the extractions of dental organs that were carried out by the Dentistry service in a Health Center of the Sierra de Zongolica that belongs to the municipality of Astacinga. Results. 599 extractions were performed, 236 primary and 363 permanent in 483 users of which 305 were women, which represented 63.1\%. Discussion: oral health as an integral part of general health is still far away, curative activity continues to be prioritized in the place of preventive activities, in the results it can be observed that people arrive late to a dental consultation with the diagnosis of retained tooth root. Conclusion: the first lower molars in the permanent dentition were the most affected while the upper anterior central incisors in the primary organs.
\end{abstract}

Keywords. (DeCS): dental extractions; frequency; cause; dental organs.

Artículo recibido: 30 noviembre. 2021 Aceptado para publicación: 29 diciembre 2021 Correspondencia: jaimejaramillo09@hotmail.com Conflictos de Interés: Ninguna que declarar 


\section{INTRODUCCIÓN}

En las últimas décadas la transición demográfica y económica, han sido factores determinantes en el cambio de los estilos de vida de la población y perfil epidemiológico de las principales causas de morbimortalidad en los países desarrollados o con economías emergentes. En México, actualmente las enfermedades crónicas son las más comunes y costosas de todos los problemas de salud, sin embargo también son las más prevenibles como la caries dental (Gobierno de México, 2014).

En el mundo se ven afectadas 3.9 mil millones de personas por enfermedades bucales, la caries dental no tratada representa casi la mitad del total de la población (44\%) lo que la convierte en la más prevalente entre 1999 y 2010 y es el cuarto gasto en salud comparado con otras enfermedades no transmisibles (ENT), (FDI World Dental Federation, 2017).

Se ha demostrado que la caries dental y las enfermedades periodontales son las principales causas de pérdida de dientes, otras incluyen pericoronitis, traumatismos, ortodoncia, prótesis y neoplasias (Osunde et al., 2017; Hupp, 2010), aunque también se han relacionado con la higiene bucal, educación, nivel socioeconómico y calidad de vida individual, las personas que presentan bajos ingresos y baja educación son más propensos a perder piezas dentales que aquellas de ingreso más alto (Taiwo et al., 2017; Cunha et al., 2015).

Eliminar focos de infección evitando daños a futuro es parte fundamental del servicio de Odontología además una mala salud oral tiene impacto sobre las personas de varias maneras: muchas condiciones causan dolor, afectan a la calidad de vida, reducen la productividad escolar y laboral. Los cuidados requeridos resultan en una pesada carga financiera para los sistemas sanitarios y para los afectados (FDI World Dental Federation, 2015, Gobierno de México, 2014).

La pérdida de dientes es considerada un indicador importante de la salud bucal de la población y tiene un fuerte impacto en el bienestar social. Sus efectos incluyen una disminución de la capacidad funcional de la masticación y el habla, así como una disminución en el estado nutricional, cambios estéticos y efectos psicológicos, con un resultado negativo, asociado en términos de autoestima e integración social (Gobierno de México, 2014)

Los dientes son órganos ectodérmicos generados por la mayoría de los vertebrados, y se producen en varios tamaños, formas y números. La mayoría de los vertebrados 
reemplazan sus órganos dentarios continuamente (polifiodontes). Con la excepción de los mamíferos tienen una capacidad de regeneración dental restringida y solo los cambian una vez (difiodontes) o no lo hacen (monofiodontes) (Balic, 2018).

En México de acuerdo con la normativa nacional define: órgano dentario del aparato estomatognático con estructura y funciones propias (NORMA Oficial Mexicana NOM013-SSA2-2015, Para La Prevención y Control de Enfermedades Bucales, 2015). Desde el primer dentista Hesi Re (3100-2181 A.C.) la primera opción de tratamiento era la extracción de órganos dentales, en 1764 los dentistas de las colonias americanas utilizaban llaves para realizar los procedimientos y a menudo los herreros eran los que hacían los procedimientos (Mitra, 2011).

La extracción dental es un procedimiento quirúrgico donde se retira un diente, en la mayoría de los casos son órganos dentarios afectados por enfermedades, provocando que alteren la función masticatoria y en ocasiones pueden poner en riesgo la salud por ser focos infecciosos (Ramírez Balderas et al., 2010). El procedimiento consiste en separar la articulación alveolo dentaria que está formada por encía, hueso, diente y ligamento periodontal, desgarrándolo en su totalidad, para conseguir luxar y extraer el diente. El procedimiento ideal es la extirpación total del diente o de la raíz dentaria con el minino de daño de los tejidos circundantes (Gay Escoda \& Berini Aytés, 2006; Jain, 2021). Es importante descartar otras opciones terapéuticas por lo que el diagnóstico juega un papel crucial, la posición del paciente y del operador debe ser lo más favorables importante que el paciente tenga la posición más favorable, contar con instrumental adecuado e instalaciones para poder realizar el procedimiento correspondiente (Martínez Treviño, 2009), anestesia, realizar anestesia de la zona de acuerdo al órgano dentario a extraer, utilizando la técnica adecuada de infiltración es importante tener en cuenta la posición del paciente y la aplicación previa de un antiséptico antes de introducir la aguja (Ojeda Suárez \& Ocegueda Estrada, 2010).

Dentro de los estudios señalan que la extracción es un método frecuente como resultado de problemas o comportamientos adoptados por el individuo (Olate et al., 2006; Saheeb \& Sede, 2013; Osaghae \& Azodo, 2014; Osunde et al., 2017; Villares López et al., 2016; Cunha et al., 2015; Ramírez Balderas et al., 2010; Vega et al., 2018) y según datos de la Dirección General de Información en Salud (DGIS) (Secretaría de Salud, 2017), perteneciente a la Secretaría de Salud en 2018 en el país se realizaron: 453,830 
extracciones en órganos primarios; 559,137 en permanentes, 72,422 cirugías bucales, para un total de 1,085389. En el estado de Veracruz en el mismo año fue de: 27,654 en órganos primarios; 45,161 en permanentes, 2,590 cirugías bucales y un total de 75,405. A nivel local en la Jurisdicción Sanitaria VII de Orizaba se realizaron: 3,025 extracciones en órganos primarios; 5,015 en permanentes con 383 cirugías bucales para un total de 8,423 (Secretaría de Salud, 2020). El municipio de Astacinga el cual pertenece a la Sierra de Zongolica en el estado de Veracruz se encuentra en la zona centro del estado con coordenadas $18^{\circ} 34^{\prime} 03^{\prime \prime}$ ' latitud norte $97^{\circ} 06^{\prime}$. A una altura de 2300 metros sobre el nivel del mar. Limita al norte con el municipio de Tlaquilpa, al noreste con Mixtla de Altamirano, al Sureste con Tehuipango y al sur y al oeste con el estado de Puebla. La distancia aproximada de Astacinga al municipio de Orizaba es de 42km (Secretaría de Finanzas y Planeación del Estado de Veracruz, 2015).

Por lo anterior, se propone como objetivo fue determinar las causas más frecuentes de extracción dental en el servicio de odontología de un Centro de Salud de la Sierra de Zongolica.

\section{ESTRATEGIAS METODOLÓGICAS}

Estudio cuantitativo descriptivo y trasversal especificando las propiedades, características y perfiles de personas, grupos, comunidades, permitiendo medir o recoger información sobre las variables a las que se refieren. Por la obtención de la información que fue de enero 2015 a diciembre 2019, es retrospectivo (Hernández Sampieri et al., 2014), con la finalidad de conocer las causas de extracción de órganos dentales en el servicio de odontología del Centro de Salud Huapango.

En relación con la población, el Centro de Salud de Huapango tiene en su área de influencia 6 localidades: Huapango, Xayacatepec, Mexcaltitla, Moyoapan, Zacatecoxco y Tlapacoyan con una población aproximada de: 1230 personas de acuerdo con el micro diagnóstico de la unidad el 95\% de la población es hablante de náhuatl.

Los criterios de selección establecidos fueron inclusión: registro de extracciones de órganos dentarios en la hoja diaria del servicio de Estomatología realizadas en hombres y mujeres, que acudieron al Centro de Salud de Huapango entre enero 2015 y diciembre 2019. Exclusión: registro de cirugías bucales de terceros molares, referencia de exodoncias a segundo nivel de atención. Eliminación: las extracciones registradas en la hoja diaria que estén incompletas en los datos. La búsqueda de los datos se realizó en las 
fuentes de información de hoja diaria de Estomatología SINBA-SIS-02-P comprendido entre enero 2015 a diciembre 2019 con 483 usuarios atendidos por extracción dental en el Centro de Salud Huapango se registró: edad, sexo, fecha de exodoncia, residencia, nomenclatura de órgano extraído, número de órganos, diagnóstico de extracción, enfermedad (diabetes, hipertensión, otra), y si estaba embarazada en el momento de la extracción dental.

Para identificar el órgano dentario se utilizó la nomenclatura dental de la FDI como la estable la NOM 013 donde cada diente se numera con dos dígitos, siendo el primero el que hace referencia al cuadrante en el que se encuentra el diente $(1,2,3$ y 4 si son dientes permanentes, y 5, 6, 7 y 8 si son temporales), y el segundo es el que se refiere al diente propiamente dicho. La numeración dentaria será de 1 a 8 en la dentición permanente y de 1 a 5 en la dentición temporal. El sistema de dos dígitos o de la FDI es muy intuitivo ya que al emplearse números arábigos no requiere traducción a otros idiomas y es fácilmente comunicable (NORMA Oficial Mexicana NOM-013-SSA2-2015, Para La Prevención y Control de Enfermedades Bucales, 2015; Castejón Navas et al., 2001).

El diagnóstico registrado en la hoja diaria se agruparon 8 grupos de acuerdo con la codificación CIE-10: Caries (relacionado con K020, K021, K022, K023, K024, K028, K029), Enfermedades periodontales (relacionado con K050, K052, K053, K055), raíz dental retenida (dientes primarios y permanentes K038), Pulpitis (K040), fractura (S032, fractura coronaria), alteraciones en la erupción dentaria (K006, K007), fines protésicos (por indicación protésica) y absceso (K046, K047, K052), (Organización Mundial de la Salud, 2008).

Los investigadores no presentaron conflictos de interés y se apega a la "Declaración de Helsinki de la Asociación Médica Mundial" esta investigación fue aprobada por el Comité de Investigación (CI) HRRB/CI/2020/42 y Comité de Ética en Investigación (CEI) HRRB/CEI/2020/43 del Hospital Regional Río Blanco. Para mantener la confidencialidad la información se registró el número de expediente y solo un investigador lo hizo, tomando los datos de la persona involucrada respetado los "Principios éticos aplicados a la epidemiología. Otro referente ético considerado en el presente estudio fueron las pautas Internacionales para la Evaluación Ética de los Estudios Epidemiológicos CIOMS" que se describe en el apartado de confidencialidad (Gobierno 
de México, 2019). El riego declarado fue nulo, debido a que la información se obtuvo de la hoja diaria de Estomatología.

Para el análisis de datos se registro en una hoja de Excel donde quedo numéricamente para después procesar en el paquete estadístico Statistical Package for the Social Sciences, IBM® SPSS® Statistics Versión 23, creando una base de datos donde se capturó la información, previa validación y revisión del llenado completo y correcto. El plan de análisis estuvo integrado por el uso de estadística descriptiva con frecuencia porcentajes, medidas de tendencia central (media, moda, mediana), y dispersión.

\section{RESULTADOS Y DISCUSIÓN}

En la edad se obtuvo un rango de 83 con una mínima de 4 y máxima de 87 , media de 28.35, mediana 22, moda de 8, desviación estándar de 21.604, varianza de 466.751. La residencia se distribuyó entre las seis localidades del área de responsabilidad del centro de salud de la siguiente manera: 148 (30.6\%) de Huapango, 66 de Moyoapan (13.7\%), 47 (9.7\%) de Mexcaltitla, 28 (5.8\%) de Xayacatepec, 7 (1.4\%) de Zacatecoxco y 7 (1.4\%) Tlapacoyan y el resto fueron foráneos 180 (37.3\%). El año con mayor número de extracciones se registró en 2015 con 168.

\section{Tabla 1}

Extracciones por año y atención por sexo

\begin{tabular}{lccccc}
\hline \multirow{2}{*}{ Año } & \multirow{2}{*}{ Extracciones } & \multicolumn{2}{c}{ Atención por sexo } & \multicolumn{2}{c}{ Total } \\
\cline { 3 - 6 } & & $\mathbf{H}$ & $\mathbf{M}$ & $\boldsymbol{f}$ & \% \\
\hline 2015 & 168 & 53 & 89 & 142 & 29.4 \\
2016 & 141 & 47 & 74 & 121 & 25.1 \\
2017 & 111 & 23 & 56 & 79 & 16.4 \\
2018 & 85 & 30 & 39 & 69 & 14.3 \\
2019 & 94 & 25 & 47 & 72 & 14.9 \\
\hline Total & $\mathbf{5 9 9}$ & $\mathbf{1 7 8}$ & $\mathbf{3 0 5}$ & $\mathbf{4 8 3}$ & 100.0 \\
\hline
\end{tabular}

Fuente: Registro en la hoja diaria de Estomatología

En la Tabla 1 se puede observar las extracciones por año y sexo, en el período de enero 2015 a diciembre 2019 se atendieron 483 personas por procedimientos de extracción dental, 178 (36.9\%) hombres y $305(63.1 \%)$ mujeres. En todos los años las mujeres fueron quien más se sometieron a una extracción. 
De 2015 a 2019, se realizaron 599 extracciones en 236 en órganos primarios y 363 en permanentes de las cuales 211 hombres, a 147 se les realizó una extracción, a 29 dos en el mismo momento y a dos 3, por otra parte, en mujeres fueron 388, a 234 se les realizó una extracción a 59 dos y a 12 tres.

\section{Tabla 2}

Relación entre diagnóstico y sexo

\begin{tabular}{lcccc}
\hline \multirow{2}{*}{ Diagnóstico } & \multicolumn{2}{c}{ Sexo } & \multicolumn{2}{c}{ Total } \\
\cline { 2 - 5 } & Hombre & Mujer & $\boldsymbol{f}$ & \% \\
\hline Caries & 24 & 69 & 93 & 19.3 \\
Enfermedad periodontal & 19 & 24 & 43 & 8.9 \\
Raíz dental retenida & 71 & 110 & $\mathbf{1 8 1}$ & $\mathbf{3 7 . 5}$ \\
Pulpitis & 15 & 41 & 56 & 11.6 \\
Fractura & 5 & 19 & 24 & 5.0 \\
Alteraciones en la erupción dentaria & 42 & 37 & 79 & 16.4 \\
Fines protésicos & 1 & 1 & 2 & .4 \\
Absceso & 1 & 4 & 5 & 1.0 \\
\hline Total & $\mathbf{1 7 8}$ & $\mathbf{3 0 5}$ & $\mathbf{4 8 3}$ & $\mathbf{1 0 0}$ \\
\hline
\end{tabular}

Fuente: Registro en la hoja diaria de Estomatología

En la Tabla 2 dentro de los diagnósticos el resultado que más se presentó fue raíz dental retenida 181 (37.5\%) hombre 71 y mujeres 110, seguido de caries con 93 (19.3\%), alteraciones en la erupción dentaria 79 (16.4\%), pulpitis 56 (11.6\%) enfermedad periodontal $43(8.9 \%)$, fractura $24(5 \%)$, absceso $5(1 \%)$ y fines protésicos $2(.4 \%)$.

\section{Tabla 3}

Extracciones por grupo de edad y sexo

\begin{tabular}{|c|c|c|c|c|}
\hline \multirow{2}{*}{ Grupo de edad } & \multicolumn{2}{|c|}{ Sexo } & \multirow{2}{*}{ Total } & \multirow{2}{*}{ Extracciones realizadas } \\
\hline & Hombre & Mujer & & \\
\hline $4-9$ & 70 & 72 & 142 & 174 \\
\hline $10-19$ & 38 & 56 & 94 & 106 \\
\hline $20-59$ & 52 & 145 & 197 & 235 \\
\hline $60-87$ & 18 & 32 & 50 & 84 \\
\hline Total & 178 & 305 & 483 & 599 \\
\hline
\end{tabular}

Fuente: Registro en la hoja diaria de Estomatología

En la Tabla 3 se observa que dentro de los grupos de edad que se le realizó la mayor cantidad de extracciones fue de 20 a 29 (235), seguido del grupo de 4 a 9 (174), 10 a 19 (106) y de 60 a 87 (84), en todos los grupos de edad se presentaron mayor cantidad de mujeres. 


\section{Tabla 4}

Dentición primaria, frecuencia por sexo, diagnóstico

\begin{tabular}{|c|c|c|c|c|c|c|c|c|c|c|c|}
\hline \multirow{2}{*}{ Órgano dentario } & \multicolumn{2}{|c|}{$f$} & \multicolumn{7}{|c|}{ Diagnóstico } & \multicolumn{2}{|c|}{ Total } \\
\hline & $\mathbf{H}$ & M & C & $\mathbf{E}$ & $\mathbf{R}$ & $\mathbf{P}$ & $\mathbf{F}$ & Al & $\mathbf{A}$ & $f$ & $\%$ \\
\hline 61 & 16 & 13 & 0 & 2 & 11 & 0 & 1 & 13 & 2 & 29 & 12.2 \\
\hline 85 & 13 & 13 & 6 & 0 & 12 & 1 & 0 & 6 & 1 & 26 & 11.01 \\
\hline 51 & 16 & 7 & 0 & 0 & 12 & 0 & 1 & 10 & 0 & 23 & 9.74 \\
\hline 81 & 9 & 12 & 0 & 2 & 1 & 0 & 0 & 18 & 0 & 21 & 8.89 \\
\hline 84 & 13 & 6 & 1 & 0 & 13 & 0 & 0 & 5 & 0 & 19 & 8.05 \\
\hline 75 & 10 & 9 & 1 & 4 & 11 & 1 & 0 & 2 & 0 & 19 & 8.05 \\
\hline 71 & 9 & 9 & 0 & 2 & 1 & 0 & 0 & 15 & 0 & 18 & 7.62 \\
\hline 55 & 3 & 10 & 1 & 1 & 6 & 1 & 0 & 4 & 0 & 13 & 5.5 \\
\hline 54 & 5 & 8 & 1 & 2 & 5 & 1 & 0 & 4 & 0 & 13 & 5.5 \\
\hline 64 & 5 & 8 & 2 & 3 & 6 & 0 & 0 & 2 & 0 & 13 & 5.5 \\
\hline 65 & 5 & 6 & 2 & 1 & 6 & 0 & 0 & 2 & 0 & 11 & 4.66 \\
\hline 74 & 2 & 6 & 1 & 0 & 6 & 0 & 0 & 1 & 0 & 8 & 3.38 \\
\hline 62 & 3 & 3 & 0 & 0 & 3 & 0 & 0 & 3 & 0 & 6 & 2.54 \\
\hline 53 & 2 & 3 & 0 & 2 & 2 & 0 & 0 & 1 & 0 & 5 & 2.11 \\
\hline 52 & 2 & 2 & 0 & 0 & 1 & 0 & 0 & 3 & 0 & 4 & 1.69 \\
\hline 63 & 1 & 3 & 0 & 2 & 1 & 0 & 0 & 1 & 0 & 4 & 1.69 \\
\hline 73 & 0 & 2 & 0 & 0 & 1 & 0 & 0 & 1 & 0 & 2 & 0.84 \\
\hline 72 & 1 & 1 & 0 & 0 & 0 & 0 & 0 & 2 & 0 & 2 & 0.84 \\
\hline Total & 115 & 121 & 15 & 21 & 98 & 4 & 2 & 93 & 3 & 236 & 100 \\
\hline
\end{tabular}

Nota: la nomenclatura del órgano dentario de acuerdo con la FDI. C: Caries, E:

Enfermedades periodontales, R: Raíz dental retenida, P: Pulpitis, F: Fractura dentaria, AL: Alteraciones en la erupción dentaria, A: Abscesos $n=236$

Fuente: Registro en la hoja diaria de Estomatología

En la Tabla 4 se aprecia que el órgano primario que con mayor frecuencia se extrajo fue el incisivo central superior izquierdo con 29 en 16 hombres y 13 mujeres que representó el $12.2 \%$ (236), seguido de segundo molar inferior con 26, incisivo central superior derecho primario con 23, los órganos que no registraron extracción fueron el incisivo lateral inferior derecho y canino inferior derecho.

El diagnóstico más frecuente en órganos primarios fue raíz dental retenida con $41.52 \%$, seguida de alteraciones en la erupción dentaria con $39.40 \%$, no se presentó ningún diagnóstico para fines protésicos. 
Tabla 5 Dentición permanente, frecuencia por sexo, diagnóstico

\begin{tabular}{|c|c|c|c|c|c|c|c|c|c|c|c|c|}
\hline \multirow{2}{*}{$\begin{array}{l}\text { Órgano } \\
\text { dentario }\end{array}$} & \multicolumn{2}{|c|}{$f$} & \multicolumn{8}{|c|}{ Diagnóstico } & \multicolumn{2}{|c|}{ Total } \\
\hline & H & $\mathbf{M}$ & $\mathbf{C}$ & $\mathbf{E}$ & $\mathbf{R}$ & $\mathbf{P}$ & $\mathbf{F}$ & Al & PR & $\mathbf{A}$ & $f$ & $\%$ \\
\hline 36 & 14 & 47 & 13 & 4 & 28 & 13 & 3 & 0 & 0 & 0 & 61 & 16.8 \\
\hline 46 & 8 & 29 & 16 & 1 & 10 & 8 & 1 & 0 & 0 & 1 & 37 & 10.1 \\
\hline 37 & 9 & 23 & 12 & 2 & 13 & 5 & 0 & 0 & 0 & 0 & 32 & 8.81 \\
\hline 16 & 4 & 17 & 8 & 0 & 9 & 2 & 2 & 0 & 0 & 0 & 21 & 5.78 \\
\hline 35 & 3 & 17 & 3 & 2 & 13 & 2 & 0 & 0 & 0 & 0 & 20 & 5.5 \\
\hline 25 & 7 & 10 & 6 & 3 & 1 & 4 & 1 & 0 & 2 & 0 & 17 & 4.68 \\
\hline 26 & 6 & 11 & 5 & 3 & 4 & 3 & 1 & 0 & 1 & 0 & 17 & 4.68 \\
\hline 15 & 4 & 10 & 2 & 0 & 6 & 4 & 2 & 0 & 0 & 0 & 14 & 3.85 \\
\hline 47 & 4 & 9 & 4 & 0 & 6 & 1 & 1 & 0 & 0 & 1 & 13 & 3.58 \\
\hline 45 & 3 & 9 & 1 & 1 & 4 & 3 & 3 & 0 & 0 & 0 & 12 & 3.3 \\
\hline 34 & 3 & 6 & 1 & 1 & 7 & 0 & 0 & 0 & 0 & 0 & 9 & 2.47 \\
\hline 44 & 2 & 7 & 0 & 0 & 5 & 3 & 1 & 0 & 0 & 0 & 9 & 2.47 \\
\hline 22 & 2 & 6 & 1 & 1 & 3 & 1 & 2 & 0 & 0 & 0 & 8 & 2.2 \\
\hline 24 & 2 & 6 & 1 & 2 & 3 & 1 & 0 & 0 & 1 & 0 & 8 & 2.2 \\
\hline 27 & 4 & 4 & 0 & 1 & 4 & 2 & 1 & 0 & 0 & 0 & 8 & 2.2 \\
\hline 48 & 2 & 6 & 4 & 0 & 4 & 0 & 0 & 0 & 0 & 0 & 8 & 2.2 \\
\hline 11 & 2 & 5 & 0 & 1 & 3 & 0 & 3 & 0 & 0 & 0 & 7 & 1.92 \\
\hline 38 & 2 & 5 & 3 & 2 & 1 & 1 & 0 & 0 & 0 & 0 & 7 & 1.92 \\
\hline 17 & 5 & 1 & 2 & 1 & 3 & 0 & 0 & 0 & 0 & 0 & 6 & 1.65 \\
\hline 13 & 2 & 4 & 0 & 0 & 6 & 0 & 0 & 0 & 0 & 0 & 6 & 1.65 \\
\hline 14 & 0 & 5 & 1 & 0 & 4 & 0 & 0 & 0 & 0 & 0 & 5 & 1.37 \\
\hline 28 & 1 & 4 & 2 & 1 & 0 & 2 & 0 & 0 & 0 & 0 & 5 & 1.37 \\
\hline 12 & 1 & 3 & 0 & 0 & 3 & 0 & 1 & 0 & 0 & 0 & 4 & 1.1 \\
\hline 23 & 0 & 4 & 1 & 0 & 3 & 0 & 0 & 0 & 0 & 0 & 4 & 1.1 \\
\hline 33 & 1 & 3 & 0 & 0 & 4 & 0 & 0 & 0 & 0 & 0 & 4 & 1.1 \\
\hline 32 & 0 & 4 & 0 & 0 & 4 & 0 & 0 & 0 & 0 & 0 & 4 & 1.1 \\
\hline 31 & 0 & 4 & 0 & 1 & 3 & 0 & 0 & 0 & 0 & 0 & 4 & 1.1 \\
\hline 18 & 2 & 1 & 1 & 1 & 1 & 0 & 0 & 0 & 0 & 0 & 3 & 0.82 \\
\hline 21 & 0 & 3 & 0 & 0 & 1 & 1 & 1 & 0 & 0 & 0 & 3 & 0.82 \\
\hline 41 & 1 & 2 & 0 & 0 & 2 & 0 & 0 & 1 & 0 & 0 & 3 & 0.82 \\
\hline 43 & 2 & 1 & 0 & 0 & 2 & 0 & 1 & 0 & 0 & 0 & 3 & 0.82 \\
\hline 42 & 0 & 1 & 0 & 0 & 1 & 0 & 0 & 0 & 0 & 0 & 1 & 0.27 \\
\hline Total & 96 & 267 & 87 & 28 & 161 & 56 & 24 & 1 & 4 & 2 & 363 & 100 \\
\hline
\end{tabular}

Nota: la nomenclatura del órgano dentario de acuerdo con la FDI. C: Caries, E:

Enfermedades periodontales, $\boldsymbol{R}:$ Raíz dental retenida, P: Pulpitis, $\boldsymbol{F}$ : Fractura dentaria,

AL: Alteraciones en la erupción dentaria, PR: Fines protésicos, A: Abscesos $n=363$

Fuente: Registro en la hoja diaria de Estomatología 
En la Tabla 5 se observa que en la dentición permanente el primer molar inferior izquierdo fue el órgano más extraído en 14 hombre 47 mujeres con un total de 61, que representó el $16.80 \%$ de las 363 extracciones dentales, seguido del primer molar inferior derecho con $37(10.1 \%)$ en 8 hombres y 29 mujeres, el incisivo lateral inferior derecho $1(0.27 \%)$ fue el menos extraído.

En cuanto al diagnóstico: raíz dental retenida representó el $44.35 \%$, seguido de caries con $23.96 \%$, pulpitis con $15.42 \%$. Del total de 483 personas registradas 11 padecen diabetes (2.3\%), hipertensión 8 (1.7\%) ninguna se registró con ambas y 464 (96.1\%) sin ninguna enfermedad.

En este estudio el objetivo fue determinar las causas más frecuente para la extracción dentaria en la localidad de Huapango perteneciente al municipio de Astacinga en la Sierra de Zongolica, el cual se encuentra entre de los municipios con menor Índice de Desarrollo Humano (IDH) en el estado con 0.545 (Gobierno del Estado de Veracruz, 2019), ya que entre menor IDH existe mayor probabilidad de extracciones dentales en las personas como lo describe (Cunha et al., 2015), por esto la atención medica incluyendo la salud bucal es muy importante para la población que vive en este municipio.

La atención primaria de la salud desde 1978 en Alma Ata (OMS/OPS/Unicef, 1978), y la salud bucal como parte integral de la salud general en Estambul 2013 (FDI World Dental Federation, 2013), aún se encuentra lejos de estarlo, se sigue priorizando la actividad curativa en lugar de actividades preventivas, la atención a las comunidades sigue en rezago, en los resultados se puede observar que las personas llegan tardíamente a consulta odontológica con el diagnóstico de raíz dental retenida (para ambas denticiones) lo que refleja que las acciones siguen siendo mutilantes.

Lo anterior, difiere con otros estudios (Ramírez Balderas et al., 2010; Osunde et al., 2017; Taiwo et al., 2017; Osaghae \& Azodo, 2014; Medina-Solís et al., 2013; Vega et al., 2018) donde la caries y sus secuelas fue el principal diagnóstico, debido a que en éste se consideró de acuerdo a la Clasificación Internacional de Enfermedades-10 (CIE-10) (Organización Mundial de la Salud, 2008) como lo establece la NORMA Oficial Mexicana NOM-013-SSA2-2015, Para la prevención y control de enfermedades bucales, en su numeral 9.2.2.11 (NORMA Oficial Mexicana NOM-013-SSA2-2015, Para La Prevención y Control de Enfermedades Bucales, 2015), por lo que no se tomó como secuela raíz dental retenida sino como un diagnóstico propio. 
A diferencia de Osunde et al., (2017) la mayor cantidad de personas que se presentaron al procedimiento fueron mujeres al igual que lo encontrado por otros autores (Ramírez Balderas et al., 2010; Gómez-Delgado \& Montero-Aguilar, 2011; Vega et al., 2018). También se observó que el órgano que más se perdía era el primer molar inferior izquierdo con $16.80 \%$ a diferencia de Taiwo et al. (2017) donde fue el tercer molar inferior izquierdo con $11.7 \%$

En la dentición primaria el diagnóstico más frecuente fue raíz dental retenida con un $41.52 \%$ seguido de alteraciones en la erupción dentaria con un $39.40 \%$ a diferencia de lo reportado por Villares López et al. (2016) donde el diagnostico más frecuente para exodoncia fue alteraciones en la erupción dentaria con $39.2 \%$

\section{CONCLUSIÓN}

Con los resultados obtenidos se puede concluir que en este estudio los órganos dentarios que con mayor frecuencia son extraídos corresponde a los primeros molares inferiores en la dentición permanente y la perdida de incisivos centrales anteriores superiores en la dentición primaria, las mujeres fue el grupo de personas que más acudió al servicio.

La pérdida de órganos dentarios afecta la calidad de vida por lo que este tipo de investigación en entornos con bajo Índice de Desarrollo Humano (IDH), puede permitir políticas públicas en beneficio de la población usuaria de las unidades de primer nivel de atención, dando prioridad a la prevención y tratar de evitar la mutilación dentaria a temprana edad.

También el diagnóstico de raíz dental retenida, nos muestra que los pacientes acuden en etapas tardías a control bucal, por lo que se puede elaborar estrategias específicas por grupo de edad, para la prevención de caries fomentando medidas preventivas y el autocuidado.

Acciones como detección de placa bacteriana, enseñanza de la técnica de cepillado y aplicación de flúor se vuelven importantes en las poblaciones más vulnerables, el monitoreo de estas acciones, así como los recursos disponibles son pieza clave en el funcionamiento del programa de salud bucal.

Dentro de las limitaciones de este estudio se encuentra la valoración de los determinantes sociales como religión, educación e higiene bucal de las personas usuarias de los servicios de salud. 


\section{LISTA DE REFERENCIAS}

Balic, A. (2018). Biology Explaining Tooth Repair and Regeneration: A Mini-Review. Gerontology, 64(4), 382-388. Recuperado de https://doi.org/10.1159/000486592

Castejón Navas, I., Magán Sánchez, R., \& García Ballesta, C. (2001). Sistema de notación dentaria. Odontol Pediátr, 9(3), 126-128. Recuperado de https://www.odontologiapediatrica.com/wp-content/uploads/2018/05/14_3.Catejon-Navas.pdf

Cunha, M. A., Lino, P. A., Santos, T. R., Vasconcelos, M., Lucas, S. D., \& Abreu, M. H. (2015). A 15-Year Time-series Study of Tooth Extraction in Brazil. Medicine(Baltimore), 94(47), e1924. Recuperado de https://www.ncbi.nlm.nih.gov/pmc/articles/PMC5058957/

FDI World Dental Federation. (2013). Oral health and general health Declaration: Istanbul declaration. FDI World Dental Internacional. Recuperado de https://www.fdiworlddental.org/sites/default/files/2021-03/Istanbul

Declaration.pdf

FDI World Dental Federation. (2015). El Desafío de las Enfermedades Bucodentales Una llamada a la acción global. Atlas de Salud. Federacion Dental Internacional. Recuperado de https://www.fdiworlddental.org/sites/default/files/202103/book_spreads_oh2_spanish.pdf

FDI World Dental Federation. (2017). Accelerating action on oral health and NCDs. NCD Alliance. Recuperado de https://www.fdiworlddental.org/resource/accelerating-action-oral-health-andncds

Gay Escoda, C., \& Berini Aytés, L. (2006). Principios básicos de la exodoncia. In Cirugía Bucal (pp. 200-225). Oceano/Ergon.

Gobierno de México. (2014). Programa de Acción Específico: Prevención, Detección y Control de los Problemas de Salud Bucal. 2013-2018. Plan Sectorial de Salud. Recuperado de https://www.gob.mx/salud/documentos/programa-de-accionespecifico-prevencion-deteccion-y-control-de-los-problemas-de-salud-bucal2013-2018

Gobierno de México. (2019). Comisión Nacional de Bioética. Comisión Nacional de Bioética. 
https://www.gob.mx/salud\%7Cconbioetica/es/articulos/normatividadinternacional?idiom $=\mathrm{es}$

Gobierno del Estado de Veracruz. (2019). Índice de Desarrollo Humano 2010 y 2015 y Capacidades Funcionales de los Municipios 2016. SEFIPLAN. Recuperado de http://www.veracruz.gob.mx/finanzas/wpcontent/uploads/sites/2/2019/10/Veracruz.-Informe-IDH-2015-e-ICFM-2016.pdf

Gómez-Delgado, A., \& Montero-Aguilar, M. (2011). Razones para la extracción de piezas dentales en la Facultad de Odontología, Universidad de Costa Rica. Odovtos - International Journal of Dental Sciences, 13, 36-41. Recuperado de https://www.redalyc.org/pdf/4995/499550297006.pdf

Hernández Sampieri, R., Fernández Collado, C., \& Baptista Lucio, M. P. (2014). Definición del alcance de la investigación a realizar: exploratoria, descriptiva, correlacional o explicativa. In Metología de la Investigación (Sexta ed, pp. 88101). McGraw-Hill / Interamericana editores s.a. de c.v.

Hupp, J. R. (2010). Principios de la exodoncia no complicada. In Cirugía oral y maxilofacial contemporánea (Quinta ed, pp. 95-127). Elsevier/Mosby.

Jain, A. (2021). Principles and Techniques of Exodontia. In Oral and Maxillofacial Surgery for the Clinician (pp. 259-299). Recuperado de https://doi.org/doi.org/10.1007/978-981-15-1346-6

Martínez Treviño, J. A. (2009). Extracción simple. In Cirugía oral y maxilofacial (pp. 103-121). El Manual Moderno.

Medina-Solís, C. E., Pontigo-Loyola, A. P., Pérez-Campos, E., Hernández-Cruz, P., \& De la Rosa-Santillana, R. (2013). Principales razones de extracción de dientes permanentes en una muestra de adultos mexicanos. Revista de Investigación Clínica, 65(2), 141-149. Recuperado de https://www.medigraphic.com/pdfs/revinvcli/nn-2013/nn132d.pdf

Mitra, G. V. (2011). Introducción a la Cirugía Oral. In Manual Ilustrado de Cirugía Oral y Maxilofacial (1st ed, pp. 1-4). Jaypee Highlights Medical Publishers.

Ojeda Suárez, C., \& Ocegueda Estrada, E. (2010). Técnicas anestésicas odontológicas. In Analgesia y anestesia en odontología (pp. 69-89). Trillas.

Olate, S., Alister, J. P., Soto, M., Alveal, R., Fuentes, J., \& Thomas, D. (2006). Extracciones e indicaciones de extracciones dentales en población rural chilena 
de 11 a 30 años. Av Odontoestomatol, 22(2), 119-124. Recuperado de https://scielo.isciii.es/pdf/odonto/v22n2/original2.pdf

OMS/OPS/Unicef. (1978). Declaracaión de ALMA-ATA. Recuperado de https://www.paho.org/hq/dmdocuments/2012/Alma-Ata-1978Declaracion.pdf

Organización Mundial de la Salud. (2008). Clasificación Estadística Internacional de Enfermedades y Problemas Relacionados con la Salud-10 (pp. 509-553). Organizacion Panamericana de la Salud. Recuperado de http://ais.paho.org/classifications/chapters/pdf/volume1.pdf

Osaghae, I. P., \& Azodo, C. C. (2014). Analysis of split tooth as an unstudied reason for tooth extraction. BMC Res Notes, 7, 630. Recuperado de https://www.ncbi.nlm.nih.gov/pmc/articles/PMC4168166/

Osunde, O. D., Efunkoya, A. A., \& Omeje, K. U. (2017). Reasons for loss of the permanent teeth in patients in Kano, north western Nigeria. J West Afr Coll Surg, 7(2), 47-64. Recuperado de https://www.ncbi.nlm.nih.gov/pmc/articles/PMC6016754/

Ramírez Balderas, F. A., Pérez Cervantes, B. A., Sánchez Rosales, C., \& Colín Cortés, E. (2010). Causas más frecuentes de extracción dental en la población derechohabiente de una unidad de medicina familiar del Instituto Mexicano del Seguro Social. Revista ADM, 67(1), 21-25. Recuperado de https://www.medigraphic.com/pdfs/adm/od-2010/od101e.pdf

Saheeb, B. D., \& Sede, M. A. (2013). Reasons and Pattern of Tooth Mortality in a Nigerian Urban Teaching Hospital. Ann Afr Med, 12(2), 110-114. Recuperado de https://www.annalsafrmed.org/article.asp?issn=1596-

$3519 ;$ year $=2013 ;$ volume $=12 ;$ issue $=2 ;$ spage $=110 ;$ epage $=114 ;$ aulast $=$ Saheeb

Secretaría de Finanzas y Planeación del Estado de Veracruz. (2015). Sistema de Información Municipal. Gobierno del Estado de Veracruz. Recuperado de http://www.veracruz.gob.mx/wp-content/uploads/sites/2/2015/05/Astacinga.pdf

Secretaría de Salud. (2017). Dirección General de Información en Salud. Recuperado de http://sinba08.salud.gob.mx/cubos/cubosis2018_plataforma_sinba.htm

Secretaría de Salud. (2020). Dirección General de Información en Salud. Dirección General de Información En Salud. Recuperado de http://www.dgis.salud.gob.mx/contenidos/basesdedatos/BD_Cubos_gobmx.html 
NORMA Oficial Mexicana NOM-013-SSA2-2015, Para la prevención y control de enfermedades bucales, (2015). Recuperado de https://www.dof.gob.mx/nota_detalle.php?codigo=5462039\&fecha=23/11/2016

Taiwo, A. O., Ibikunle, A. A., Braimah, R. O., Sulaiman, O. A., \& Gbotolorun, O. M. (2017). Tooth extraction: Pattern and etiology from extreme Northwestern Nigeria. Eur J Dent, 11(3), 335-339. Recuperado de https://doi.org/10.4103/ejd.ejd_160_17

Vega, V. S., Ramírez, V. M., \& Bernal, S. S. (2018). Riesgo de complicaciones a corto plazo asociadas con extracción dental en pacientes de una unidad de medicina familiar de Acapulco, Guerrero, México. Aten Fam, 25(4), 151-154. Recuperado de

https://www.medigraphic.com/cgibin/new/resumen.cgi?IDARTICULO=82949

Villares López, D. E., Rodríguez Carravilla, A. J., Ruiz Duque, C., \& Romero Maroto, M. (2016). Análisis de las causas de exodoncia en la población infantil atendida en una clínica universitaria. Rev Pediatr Aten Primaria, 18, e73-e79. Recuperado de https://scielo.isciii.es/scielo.php?script=sci_arttext\&pid=S113976322016000200006 